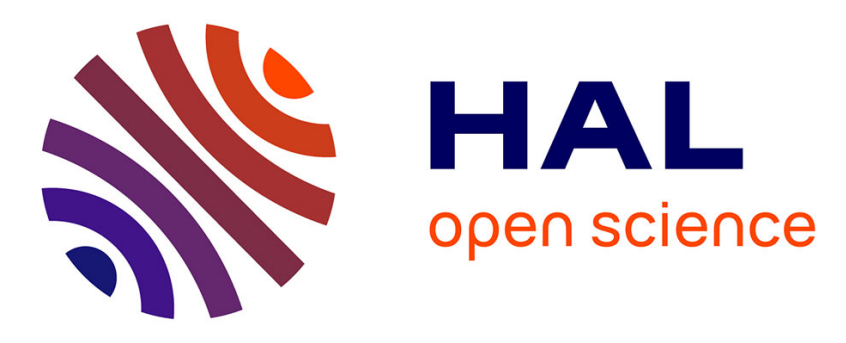

\title{
Transfert radiatif dans NO3K fondu et la fonte vitreuse de $\mathrm{B} 2 \mathrm{O} 3$
}

\author{
Tan He Ping, M. Ferre, M. Lallemand
}

\section{To cite this version:}

Tan He Ping, M. Ferre, M. Lallemand. Transfert radiatif dans NO3K fondu et la fonte vitreuse de B2O3. Revue de Physique Appliquée, 1987, 22 (2), pp.125-138. 10.1051/rphysap:01987002202012500 . jpa-00245524

\section{HAL Id: jpa-00245524 https://hal.science/jpa-00245524}

Submitted on 1 Jan 1987

HAL is a multi-disciplinary open access archive for the deposit and dissemination of scientific research documents, whether they are published or not. The documents may come from teaching and research institutions in France or abroad, or from public or private research centers.
L'archive ouverte pluridisciplinaire HAL, est destinée au dépôt et à la diffusion de documents scientifiques de niveau recherche, publiés ou non, émanant des établissements d'enseignement et de recherche français ou étrangers, des laboratoires publics ou privés. 
Classification

Physics Abstracts

$44-40$

\title{
Transfert radiatif dans $\mathrm{NO}_{3} \mathrm{~K}$ fondu et la fonte vitreuse de $\mathrm{B}_{2} \mathrm{O}_{3}$
}

\author{
Tan He Ping, M. Ferre et M. Lallemand \\ LESTE UA 1098 (CNRS), Laboratoire de Thermique, ENSMA, Rue Guillaume VII, 86021 Poitiers Cedex, \\ France
}

(Reçu le 5 mai 1986, révisé le 10 septembre, accepté le 23 octobre 1986)

\begin{abstract}
Résumé. - On étudie le transfert d'origine radiative dans des couches semi-transparentes de $\mathrm{NO}_{3} \mathrm{~K}$ fondu et $\mathrm{B}_{2} \mathrm{O}_{3}$ à l'état de fonte vitreuse. La partie expérimentale du travail a permis d'obtenir en valeur absolue les spectres d'absorption de $\mathrm{NO}_{3} \mathrm{~K}$ et $\mathrm{B}_{2} \mathrm{O}_{3}$ entre 1 et $6,3 \mu \mathrm{m}$ à partir de mesure de l'émissivité apparente de couches planes de différentes épaisseurs déposées sur un substrat métallique réflecteur. A l'aide de ces résultats la modélisation du transfert couplé conduction-rayonnement dans le problème du mur a été obtenue par l'analyse nodale. Un modèle de spectre d'absorption à 16 bandes rectangulaires a été considéré pour $\mathrm{NO}_{3} \mathrm{~K}$ et à 11 bandes pour $\mathrm{B}_{2} \mathrm{O}_{3}$ et les propriétés spectrales des frontières ont été prises en compte. Ce modèle montre la transformation spatiale des spectres de flux radiatifs à l'intérieur du mur. Le calcul de la conductivité radiative effective indique l'importance de l'effet d'épaisseur. Les résultats de la modélisation ont été appliqués pour déterminer la correction radiative à effectuer dans les mesures de conductivité thermique des sels fondus et des fontes de verre à haute température. On montre qu'il est nécessaire de considérer l'émissivité spectrale des frontières. La prise en compte de ces effets indique une influence très nette du rayonnement dans le transfert couplé dans des couches de $\mathrm{NO}_{3} \mathrm{~K}$ et $\mathrm{B}_{2} \mathrm{O}_{3}$ confinées entre des surfaces chromées ou de platine. Pour ces deux composés le calcul de la composante radiative a été effectué pour les configurations rencontrées dans différents thermo-conductimètres utilisés.
\end{abstract}

\begin{abstract}
Radiative transfer in semi-transparent slabs of molten $\mathrm{NO}_{3} \mathrm{~K}$ and vitreous melt of $\mathrm{B}_{2} \mathrm{O}_{3}$ is studied. In the experimental part, absorption spectra in absolute values are carried out between 1 and $6.3 \mu \mathrm{m}$, by means of an emittance method for various thickness of the material deposited on a metallic substratum. Modelization by nodal analysis of the combined conductive-radiative heat transfer in a slab of molten salts has been performed for spectrally selective boundaries and multiboxes models for the absorption spectrum of both substances. The model is able to calculate the spectral evolution of the radiative flux within the wall. It is proceeded to the radiative correction for the thermal conductivity data of molten salts and glass melts at high temperature. It is shown that the radiative participation with in $\mathrm{NO}_{3} \mathrm{~K}$ and $\mathrm{B}_{2} \mathrm{O}_{3}$ slabs is sensitive to the spectral emisivity of the boundaries. For molten $\mathrm{NO}_{3} \mathrm{~K}$ and vitreous $\mathrm{B}_{2} \mathrm{O}_{3}$ calculation have been effectuated for various configurations of usual thermo-conductimeters by varying thickness, spectral emissivities of boundary and temperatures difference between them.
\end{abstract}

\section{Introduction.}

Les milieux semi-transparents (MST) échangent leur énergie par la coopération des trois modes de transfert en interdépendance, de sorte qu'à un déséquilibre thermique provoqué (échelon de température, gradient de température) est associée une réponse globale du système mettant en jeu des transferts combinés masquant les processus élémentaires. A cet égard le couplage des modes conductif et radiatif est considéré comme une des principales causes d'erreur entachant les données de conductivité thermique de MST notamment dans le domaine des hautes températures.

En l'absence de formulation ou de quantification numériques précises, les expérimentateurs s'efforcent de mettre en cuvre des méthodes pour obvier à cet effet. On limite le transfert radiatif en construisant le conductimètre au moyen de métaux peu émissifs dans l'infrarouge et aux états de surface soignés. En stationnaire, l'étude peut être menée en fonction de l'épaisseur de la couche ou du gradient thermique appliqué ; les conductivités thermiques sont alors obtenues comme extrapolation des résultats vers des épaisseurs ou des gradients nuls. Des formules de correction ont également été proposées en particulier par Poltz [1], Arpaci et Larsen [2], approximations toutes deux restreintes de la théorie du transfert radiatif dans une lame plane. Le plus souvent les corrections radiatives sont appliquées de manière caricaturale se résumant à l'emploi de la conductivité radiative de Rosseland, valable seulement dans les milieux optiquement très épais, ou 
encore à l'expression du flux dans les milieux optiquement minces.

Nous nous proposons de fournir ici, dans le cas du problème du mur plan, les valeurs du flux radiatif aux parois et à l'intérieur même de la substance, obtenues par la modélisation des échanges bi-mode, conductifradiatif. La méthode est appliquée au couplage dans deux fluides typiques semi-transparents : le nitrate de potassium et le trioxyde de dibore, portés à haute température, constituant un mur limité par des frontières d'émissivités hémisphériques diffuses non grises quelconques et soumis à des gradients de température variés.

Cette étude prolonge donc celle déjà entreprise sur le couplage radiation-conduction dans les verres solides chauds qui a permis l'identification de leur conductivité thermique en présence de transfert couplé [3,4].

$\mathrm{Du}$ point de vue métrologique la consultation des articles de synthèse [5] et [6], ainsi que les articles récents sur la conductivité thermique des Nitrates, Nitrites de Potassium et de Sodium, de Santini et al. [7] ainsi que de Tufeu et al. [8] permettent de constater que pour ce nombre de transport les incertitudes demeurent grandes (de l'ordre de $40 \%$ vers $420^{\circ} \mathrm{C}$ ) ; le sens de l'effet éventuel de la température est controversé (pente positive pour [7], insensibilité à la température entre $335^{\circ} \mathrm{C}$ et $450^{\circ} \mathrm{C}$ selon [8]). Il en est de même pour $\mathrm{B}_{2} \mathrm{O}_{3}$, le désaccord entre les différents auteurs s'amplifiant à mesure qu'on s'élève en température ; ainsi à $1270 \mathrm{~K}$ les mesures de Creffield et al. [9] et celles de Lezhenin et al. [10] diffèrent de $70 \%$.

Cette dispersion des données de conductivité thermique, que l'on retrouve pour tous les sels semi-transparents ou les fontes vitreuses, est une gêne évidente, dans les applications mettant en œuvre ces milieux, mais également pour le physicien intéressé à la théorie du transport de chaleur.

La modélisation du transfert combiné est un outil puissant et fiable ; cependant la valeur de ces résultats reste subordonnée à la connaissance des spectres d'absorption des MST considérés, dans toute l'étendue du domaine spectralement utile du point de vue des échanges radiatifs. Or le plus souvent les valeurs absolues $\left(\mathrm{en} \mathrm{m}^{-1}\right.$ ) des spectres d'absorption ne sont pas connues; les informations dont on dispose, dans l'exemple des sels, concernent principalement l'identification des pics fondamentaux en spectrographie d'émission infrarouge et Raman d'intérêt physico-chimique ; leurs amplitudes restent inconnues, particulièrement dans les zones de semi-transparence.

La partie expérimentale de ce travail a donc consisté à obtenir les spectres d'absorption de $\mathrm{NO}_{3} \mathrm{~K}$ et $\mathrm{B}_{2} \mathrm{O}_{3}$ entre 1 et $6,3 \mu \mathrm{m}$ à partir de mesures directes d'absorption et d'émissivité effectuées à des températures supérieures à celle de la fusion $\left(\mathrm{NO}_{3} \mathrm{~K}\right)$ ou de la transition vitreuse $\left(\mathrm{B}_{2} \mathrm{O}_{3}\right)$.

Nous exposons dans une seconde partie la méthode expérimentale mise en œuvre pour déterminer les spectres d'absorption de $\mathrm{NO}_{3} \mathrm{~K}$ et $\mathrm{B}_{2} \mathrm{O}_{3}$ dans la région précédant les bandes d'absorption fondamentales; dans une troisième partie nous donnons les résultats de la modélisation du transfert couplé du problème du mur plan parallèle par l'analyse nodale et montrons l'influence respective de l'émissivité spectrale des frontières, de l'épaisseur de la couche ainsi que de la température du milieu. Nous utilisons finalement ces résultats pour déterminer la correction radiative à effectuer lors de l'utilisation des données de conductivité thermique de $\mathrm{NO}_{3} \mathrm{~K}$ et $\mathrm{B}_{2} \mathrm{O}_{3}$ de la littérature.

\section{Spectre d'émission de $\mathrm{NO}_{3} \mathrm{~K}$ liquide et de la fonte vitreuse de $\mathrm{B}_{2} \mathrm{O}_{3}$.}

La détermination du spectre d'absorption de sels tels que $\mathrm{NO}_{3} \mathrm{~K}$ et de fonte vitreuse comme $\mathrm{B}_{2} \mathrm{O}_{3}$ est une opération délicate en spectroscopie d'absorption à partir de $2,5 \mu \mathrm{m}$; au-delà de cette longueur d'onde on se trouve en effet dans l'obligation de s'équiper de fenêtres optiques constituées en matériau (autre que la silice fondue) pouvant supporter de hautes températures sans altération et non susceptibles d'être endommagées au moment du refroidissement, notamment par la cristallisation. Tenney et Wong [11] ont eu recours à cette méthode en préparant des films extrêmement minces de $\mathrm{B}_{2} \mathrm{O}_{3}$ recouverts de silice. Grenberg et al. [12] ont réalisé des spectres de transmission en maintenant le sel liquide par capillarité sur les mailles d'une grille. La spectroscopie de réflexion est plus aisée à mettre en œuvre [13]. Dans notre cas la spectroscopie d'absorption n'a été employée que pour $\mathrm{NO}_{3} \mathrm{~K}$ entre 1 et $2,4 \mu \mathrm{m}$; pour les longueurs d'onde plus grandes, la spectroscopie d'émission nous a paru plus adaptée. Elle a déjà été utilisée dans l'intervalle $3000-50 \mathrm{~cm}^{-1}$ par J. B. Bates et G. E. Boyd [14] au moyen d'un spectromètre à transformée de Fourier pour identification des bandes d'absorption des nitrates et des nitrites alcalins et de leur mélange, ainsi que par Kusabiraki et $Y$. Shiraishi [15] pour l'étude des silicates vitreux. Elle offre de multiples avantages: ne nécessiter aucun support transparent, s'effectuer à haute température, travailler avec des milieux préservés de l'humidité $\left(\mathrm{B}_{2} \mathrm{O}_{3}\right.$ est extrêmement hygroscopique) et dans des conditions de bonne qualité optique ; elle permet en outre de faire varier l'épaisseur de la couche à volonté.

Dans son principe, la méthode de mesure du spectre d'émission est la suivante : le liquide est déposé dans une coupelle plate en or $\left(\mathrm{NO}_{3} \mathrm{~K}\right)$ ou en platine $\left(\mathrm{B}_{2} \mathrm{O}_{3}\right)$ dont le fond est soigneusement poli, son épaisseur est déterminée avant et après l'expérience. La coupelle est disposée à l'entrée du four vertical (Fig. 1) sur un support métallique comportant un thermocouple de mesure ; la partie émissive du four est recouverte par un écran en acier inoxydable poli refroidi par une circulation d'eau et possédant une ouverture conique ne laissant apparaître que la surface libre du liquide étudié. Le four d'expérience, ainsi qu'un corps noir, sont solidaires d'un chariot manœu- 


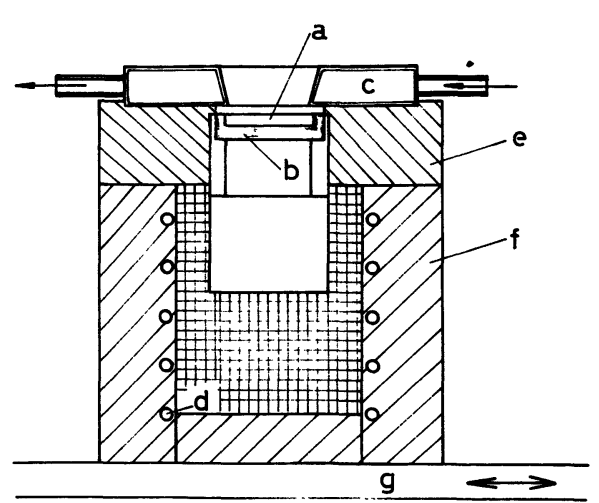
a: échantillon
b: support de platine
c: diaphragme refroidi
d: élement chauffant
e. $f$ : isolant thermique
g: support mobil

Fig. 1. - Dispositif expérimental de mesure des émissivités apparentes de couches de sels fondus ou de fontes de verres.

[Experimental set up for apparent emissivity measurements of molten salts and glassy melts.]

vrable horizontalement; les rayonnements émis par l'éprouvette et celui du corps noir sont réfléchis par un miroir à l'or et modulés. Les émisions sont alternativement détectées par un monochromateur Perkin-Elmer modèle $112 \mathrm{G}$ à faisceau unique, utilisé en simple passage, et sur lequel est adapté un télescope infrarouge à montage Cassegrain d'ouverture $50 \mathrm{~mm}$. Les signaux sont traités par une détection synchrone EG \& G 5206 et stockés dans un enregistreur de données EG \& G 4102. Dans ces conditions, lorsque la température du corps noir est exactement celle du liquide en expérience, le rapport signal couche émissive/signal corps noir, fournit l'émissivité apparente du système formé par la couche liquide et son support.

La précision de la méthode dépend de la détermination de la température de la couche qui peut se faire de deux façons; soit par mesure directe à l'aide d'un ensemble de thermocouples plongeant dans le liquide, soit par mesure radiométrique. La mesure directe est difficile pour plusieurs raisons : la lame liquide est siège d'un transfert combiné, de sorte que les thermocouples dans une couche semi-transparente n'indiquent pas correctement la température locale, étant chauffés à distance par le rayonnement (c'est le phénomène qu'on tente de mettre en évidence) ; comme la couche est relativement mince (entre 0,5 et $1 \mathrm{~mm}$ ), et que les couples doivent être disposés à la périphérie de la coupelle afin que leur rayonnement propre ne se superpose pas à l'émission du liquide, ils ressentent donc l'influence de la température des grandes masses proches. Enfin la température de la couche n'est pas uniforme étant soumise à des gradients thermiques importants notamment provoqués par les échanges convectifs à la surface libre. Les mesures effectuées à l'aide de thermocouples ont montré une incertitude d'une quinzaine de degrés à $600^{\circ} \mathrm{C}$ ce qui correspond dans le domaine spectral étudié à une variation de l'ordre de $\pm 6 \%$ sur la luminance du corps noir.

Aussi nous avons préféré opérer par voie radiométrique, qui outre l'avantage d'une grande souplesse, simplifie considérablement la construction de la cellule de mesure ; ce mode opératoire introduit cependant un certain nombre d'erreurs. En effet, dans notre cas la méthode optique de détermination de la température dépend d'une émissivité de référence. Nous l'avons choisie dans la zone d'opacité ; dans ces conditions, la température d'une couche est déterminée une fois pour toutes par l'émission des régions proches de la surface, donc plus froides que celle au voisinage du substrat, de sorte que si la détermination de la température est correcte pour les longueurs d'onde du domaine d'opacité, la méthode surévalue celle des zones de transparence influencées par la proximité du support métallique émissif.

La détermination du coefficient d'absorption $\kappa_{\lambda}\left(\mathrm{m}^{-1}\right)$ des MST étudiés a été effectuée de la façon suivante :

- pour la région spectrale comprise entre 2,3 et $6,3 \mu \mathrm{m}$ la spectrométrie d'émission a été utilisée. Dans ce cas, l'émissivité monochromatique apparente d'une couche de fluide d'épaisseur $d$ déposée sur un substrat métallique réflecteur est donnée par l'expression :

$$
E_{\lambda}(d)=\frac{\left(1-\rho_{\lambda 1}\right)\left(1-\rho_{\lambda 2} \mathrm{e}^{-2 \kappa_{\lambda} d}\right)}{\left(1-\rho_{\lambda 1} \rho_{\lambda 2} \mathrm{e}^{-2 \kappa_{\lambda} d}\right)} .
$$

A la longueur d'onde $\lambda, E_{\lambda}(d)$ dépend donc des facteurs de réflexion $\rho_{\lambda 1}$ et $\rho_{\lambda 2}$ correspondant respectivement à l'interface liquide-air (mal connue dans le proche infrarouge pour $\mathrm{NO}_{3} \mathrm{~K}$ et connue seulement dans le visible pour $\mathrm{B}_{2} \mathrm{O}_{3}$ ) et liquide-métal (connue dans tout le spectre). Afin de s'affranchir des incertitudes sur $\rho_{\lambda 1}, \kappa_{\lambda}$ a été calculé à partir de deux mesures d'émissivités apparentes $E_{\lambda}\left(d_{1}\right)$ et $E_{\lambda}\left(d_{2}\right)$ obtenues pour deux épaisseurs nettement distinctes placées dans des conditions de températures identiques.

Pour ce faire au moyen d'une couche épaisse $d_{1}$, supérieure au millimètre, formée dans la coupelle réfléchissante, on a effectué un spectre complet dans le domaine des longueurs d'onde correspondant à une absorption forte de la couche (éloignée cependant des bandes de vibrations fondamentales). Dans cette région l'absorption est considérée comme suffisamment grande pour que l'émissivité apparente de la couche donnée par (1), se réduise au seul terme $\left(1-\rho_{\lambda 1}\right)$ que l'on a pris égal à 0,97 d'après les données de Wilmshursit [13] pour $\mathrm{NO}_{3} \mathrm{~K}$ et de Prod'homme [16] pour $\mathrm{B}_{2} \mathrm{O}_{3}$. A une longueur d'onde de référence située dans ce domaine (typiquement 5,7 $\mu \mathrm{m}$ pour $\mathrm{NO}_{3} \mathrm{~K}$ ) on ajuste la température du corps noir de manière à ce que l'émissivité de la couche corresponde effectivement à cette valeur. On effectue une opération identique ensuite avec une couche plus mince d'épaisseur $d_{2} \sim d_{1} / 2$. Une vérifica- 
tion de l'égalité des températures des couches épaisses et minces est effectuée en comparant entre elles les valeurs de $\kappa_{\lambda}$ calculées à partir de (1) pour une longueur d'onde correspondant à un domaine de semitransparence pour lequel $\rho_{\lambda 1}$ peut être considéré comme connu.

Le coefficient d'absorption est ensuite calculé pour chaque longueur d'onde à partir des valeurs de $E_{\lambda}\left(d_{1}\right)$ et $E_{\lambda}\left(d_{2}\right)$ par résolution du système algébrique non linéaire en $\rho_{\lambda 1}$ et $\kappa_{\lambda}$.

- Dans l'infrarouge proche, c'est-à-dire entre 1 et $2,4 \mu \mathrm{m}$, le coefficient d'absorption a été calculé pour $\mathrm{B}_{2} \mathrm{O}_{3}$ directement à partir de la formule (1) avec un facteur de réflexion $\rho_{\lambda 1}$ pris dans [16]. Dans le cas de $\mathrm{NO}_{3} \mathrm{~K}$ (peu émissif dans ce domaine à $673 \mathrm{~K}$ ) il a été obtenu en spectroscopie d'absorption à l'aide d'une cellule en silice fondue d'épaisseur déterminée.

Le raccordement des résultats entre les deux régions spectrales a été trouvé satisfaisant.

Le facteur de réflexion de l'interface liquide-métal $\rho_{\lambda 2}$ dans le proche infrarouge est une fonction de la longueur d'onde par l'intermédiaire des valeurs des constantes optiques du métal et du liquide (voir note [17]), il a été choisi dans la référence [18].

Nous pouvons estimer dans la région $1-2,4 \mu \mathrm{m}$ que le spectre d'absorption est obtenu avec une erreur, en absorption de $\pm 2 \mathrm{~m}^{-1}$, en émission de $\pm 20 \mathrm{~m}^{-1}$; entre 2,4 et $6,3 \mu \mathrm{m}$, l'incertitude varie avec la longueur d'onde, elle est de $\pm 200 \mathrm{~m}^{-1}$ entre 2,4 et $4 \mu \mathrm{m}$ et de $\pm 100 \mathrm{~m}^{-1}$ au-delà.

\section{Spectre d'absorption de $\mathrm{NO}_{3} \mathrm{~K}$ et $\mathrm{B}_{2} \mathrm{O}_{3}$.}

La provenance des composés chimiques expérimentés est la suivante : $\mathrm{B}_{2} \mathrm{O}_{3}$, Merck Anhydre pour analyse ; $\mathrm{NO}_{3} \mathrm{~K}$, Prolabo RP Normapur.

Trioxyde de bore. La figure 2 illustre, entre 1 et $6,3 \mu \mathrm{m}$ la variation de l'émissivité apparente de deux couches de $\mathrm{B}_{2} \mathrm{O}_{3}$ d'épaisseurs respectives 0,96 et $0,47 \mathrm{~mm}$. On constate que cette région spectrale est constituée d'une zone de faible émissivité correspondant à la région de transparence qui s'étend entre 1 et $1,6 \mu \mathrm{m}$, suivie d'une zone d'émission modérée jusqu'à $2,6 \mu \mathrm{m}$; on observe la présence d'un pic intense situé à $2,78 \mu \mathrm{m}$ attribué à la présence de groupes $\mathrm{OH}$ libres ; après une seconde zone de semi-transparence, on rentre dans une zone de forte émittance correspondant aux modes d'étirement des liaisons $\mathrm{B}-\mathrm{O}$, dont les bandes d'absorption sont perceptibles sur la couche la plus mince de la figure 2 et répertoriées dans le tableau I.

La position du pic est en bon accord avec les résultats de Brohead et Newman [19] obtenus à partir de films minces élaborés dans des conditions de dessiccation drastiques. On peut noter incidemment l'absence d'autres pics d'émission attribuables soit, aux groupes $\mathrm{OH}$ de surface, soit, internes, qui modifient radicalement le spectre observable à température ambiante,

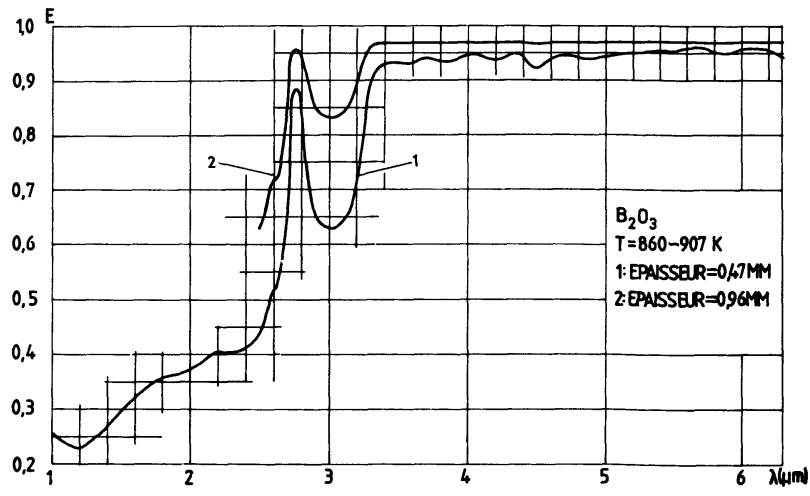

Fig. 2. - Spectre de l'émissivité monochromatique, apparente de $\mathrm{B}_{2} \mathrm{O}_{3}$ pour deux épaisseurs de matériau à $900 \mathrm{~K}$, déposées sur un support de platine.

[Apparent emissivities spectra of 2 specimens of $\mathrm{B}_{2} \mathrm{O}_{3}$ at $900 \mathrm{~K}$, deposited on a platinum substratum.]

illustrant l'intérêt méthodologique de la spectroscopie d'émission.

Le spectre d'absorption correspondant aux mesures est représenté sur la figure 3 . On y retrouve les deux zones de semi-transparence de part et d'autre du pic à $2,78 \mu \mathrm{m}$, la seconde étant centrée autour de $3 \mu \mathrm{m}$. A partir de $3,5 \mu \mathrm{m}$ le coefficient d'absorption étant de l'ordre de $3500 \mathrm{~m}^{-1}$, on rentre, du point de vue transfert radiatif, dans la région d'opacité pour les épaisseurs courantes de matériaux.

Nitrate de potassium. L'évolution spectrale de l'émissivité de deux couches de $\mathrm{NO}_{3} \mathrm{~K}$ d'épaisseur 1,73 et $0,59 \mathrm{~mm}$ est représentée sur la figure 4 et le spectre d'absorption de $\mathrm{NO}_{3} \mathrm{~K}$ déduit des mesures d'absorptance et d'émissivité apparente effectuées à $670 \mathrm{~K}$ est présenté sur la figure 5 (où les valeurs $\kappa_{\lambda}$ supérieures à $4000 \mathrm{~m}^{-1}$ sont seulement indicatives). Pour ce composé, l'émissivité de référence a été choisie à $5,7 \mu \mathrm{m}$ et le facteur de réflexion $\rho_{\lambda 1}$ a été calculé sur la base des constantes optiques de Wilmshurslt [13] ainsi que des données de dispersion de la réfractivité molaire de Gustafsson [20] déterminées à $693 \mathrm{~K}$, extrapolée au moyen de la formule :

$$
\frac{n^{2}-1}{n^{2}+2}=\frac{1,9317 \times 10^{9}}{8,2672 \times 10^{9}-\nu^{2}}
$$

où $\nu$ est la fréquence de la radiation.

Le spectre de $\mathrm{NO}_{3} \mathrm{~K}$ dans le proche infrarouge débute par une région de quasi-transparence qui s'étend jusqu'à $2,4 \mu \mathrm{m}$; il se poursuit par une série de larges bandes d'absorption intenses. Les positions de ces bandes dans la région de 2,4-6,3 $\mu \mathrm{m}$ et les régions de semi-transparence ont été reportées sur le tableau I ainsi que leur identification du point de vue du spectre de vibration de l'ion $\mathrm{NO}_{3}^{-}$. Elles sont en bon accord avec celles obtenues par Bates et Boyd [14].

Une mesure de l'efficacité du transfert radiatif pour une épaisseur de MST isotherme est fournie par le 
Tableau I. - Fréquence des maxima et minima des bandes d'émission de $\mathrm{NO}_{3} \mathrm{~K}$ et $\mathrm{B}_{2} \mathrm{O}_{3}$ entre 1 et $6,5 \mu \mathrm{m}$.

Maxima and minima frequencies emissions bands of $\mathrm{NO}_{3} \mathrm{~K}$ and $\mathrm{B}_{2} \mathrm{O}_{3}$ between 1 and $6,5 \mu \mathrm{m}$

\begin{tabular}{|c|c|c|c|c|c|c|}
\hline \multicolumn{4}{|c|}{$\mathrm{NO}_{3} \mathrm{~K}$} & \multicolumn{3}{|c|}{$\mathrm{B}_{2} \mathrm{O}_{3}$} \\
\hline $\operatorname{maxi}\left(\mathrm{cm}^{-1}\right)$ & Littérature $\left({ }^{1}\right)$ & Désignation & $\operatorname{mini}\left(\mathrm{cm}^{-1}\right)$ & $\operatorname{maxi}\left(\mathrm{cm}^{-1}\right)$ & Littérature $\left({ }^{2}\right)$ & $\operatorname{mini}\left(\mathrm{cm}^{-1}\right)$ \\
\hline 4030 & & & 3920 & & & \\
\hline 3790 & & & & 3600 & 3580 & \\
\hline & & & 3570 & & 3220 & \\
\hline 3425 & & & & & 2720 & \\
\hline & & & 3225 & & 2530 & \\
\hline 2755 & 2750 & $2 v_{3}$ & 2560 & & 2390 & \\
\hline 2380 & 2400 & $v_{1}+v_{2}$ & & & 2230 & \\
\hline & & 1 & 2210 & & 2060 & \\
\hline 2075 & 2070 & $2 v_{1}$ & & & & \\
\hline & & & $\begin{array}{l}1930 \\
1870\end{array}$ & & 1835 & \\
\hline 1750 & 1755 & $v_{1}+v_{4}$ & 1670 & & & \\
\hline
\end{tabular}

( $\left.{ }^{1}\right)$ Reference [14].

$\left({ }^{2}\right)$ Reference [19].

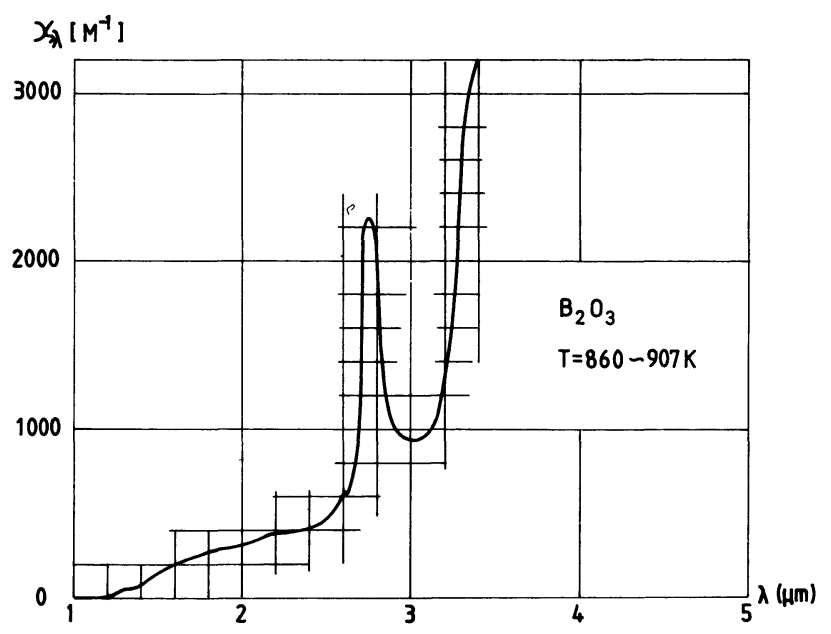

Fig. 3. - Spectre du coefficient monochromatique d'absorption de $\mathrm{B}_{2} \mathrm{O}_{3}$ à $900 \mathrm{~K}$.

[Absorption spectra of $\mathrm{B}_{2} \mathrm{O}_{3}$ at $900 \mathrm{~K}$.]

produit de l'émissivité de la couche par la fonction de Planck à la température correspondante. Nous avons représenté sur la figure 6 la variation spectrale de cette quantité pour le cas d'une lame de nitrate de potassium d'épaisseur $0,5 \mathrm{~mm}$ portée à $693 \mathrm{~K}$. Avec Bates et Boyd, nous avons admis que l'absorption au-dessus de $6,3 \mu \mathrm{m}$ est suffisamment élevée pour que l'émission soit quasi noire dans ce domaine spectral.

Deux contributions, complémentaires, gouvernent le

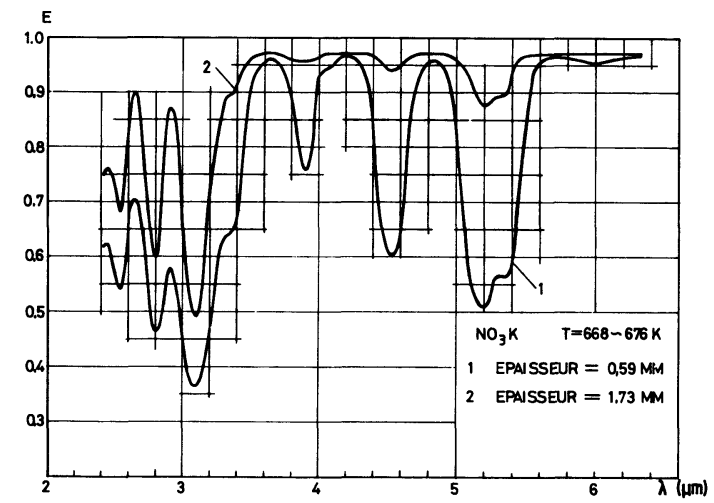

Fig. 4. - Emissivité apparente spectrale de deux couches de $\mathrm{NO}_{3} \mathrm{~K}$ déposées sur un support d'or.

[Spectral apparent emissivities of two different slabs of $\mathrm{NO}_{3} \mathrm{~K}$ deposited on gold support.]

transfert dans la couche : l'émission des surfaces, contrôlée par les bandes de semi-transparence, l'émission interne du milieu, déterminée par les bandes d'absorption. Dans une situation anisotherme le jeu de ces deux facteurs contribue à assurer le transfert à l'intérieur de la couche: l'émission des frontières, atténuées par l'absorption, l'émission locale de rayonnement de la substance à la température du milieu ; la figure 6 donne dans le cas de $\mathrm{NO}_{3} \mathrm{~K}$ pour chacun d'eux leur représentation spectrale. 


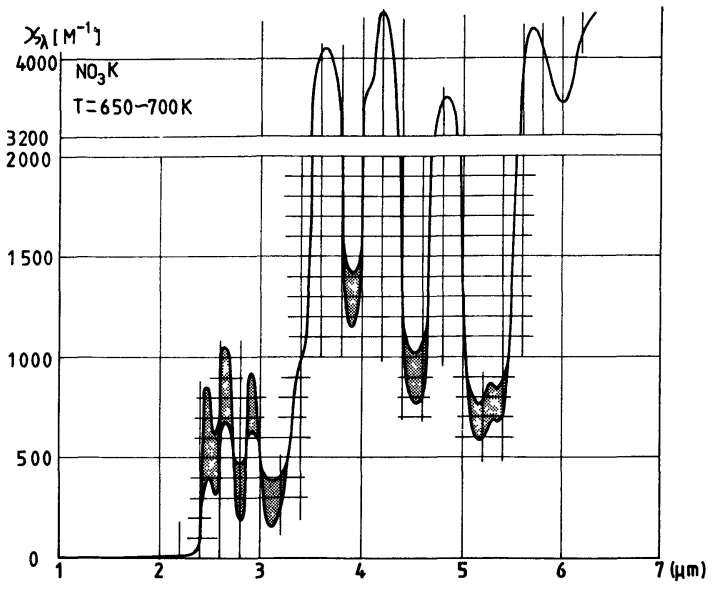

Fig. 5. - Spectre du coefficient monochromatique d'absorption de $\mathrm{NO}_{3} \mathrm{~K}$ fondu à $693 \mathrm{~K}$. Les valeurs au-dessus de $4000 \mathrm{~m}^{-1}$ sont indicatives.

[Absorption spectra of $\mathrm{NO}_{3} \mathrm{~K}$ at $693 \mathrm{~K}$ values above $4000 \mathrm{~m}^{-1}$ are indicative.]

\section{Analyse radiative.}

4.1 PRINCIPE. - Comme en [3] le calcul du flux radiatif échangé dans une lame semi-transparente en situation anisotherme est obtenu par l'analyse nodale comme résultat de la modélisation du transfert couplé.

On considère donc le cas d'un mur plan parallèle d'extension infini émettant et absorbant limité par deux frontières à réflexion diffuse d'émissivité hémisphérique spectrale $\varepsilon_{\lambda 1}=1-\rho_{\lambda 1}, \varepsilon_{\lambda 2}=1-\rho_{\lambda 2}$. Les températures de parois étant fixées et différentes, le système est le siège d'échanges conductif et radiatif.

Le calcul de la densité de flux radiatif local provenant de l'échange entre les parois d'une part, le milieu et les parois d'autre part, enfin dans le milieu lui-même, nécessite plusieurs étapes que nous rappelons brièvement.

a) On effectue une modélisation des caractéristiques optiques du système, indice de réfraction et spectre d'absorption, émissivité des frontières, à partir de

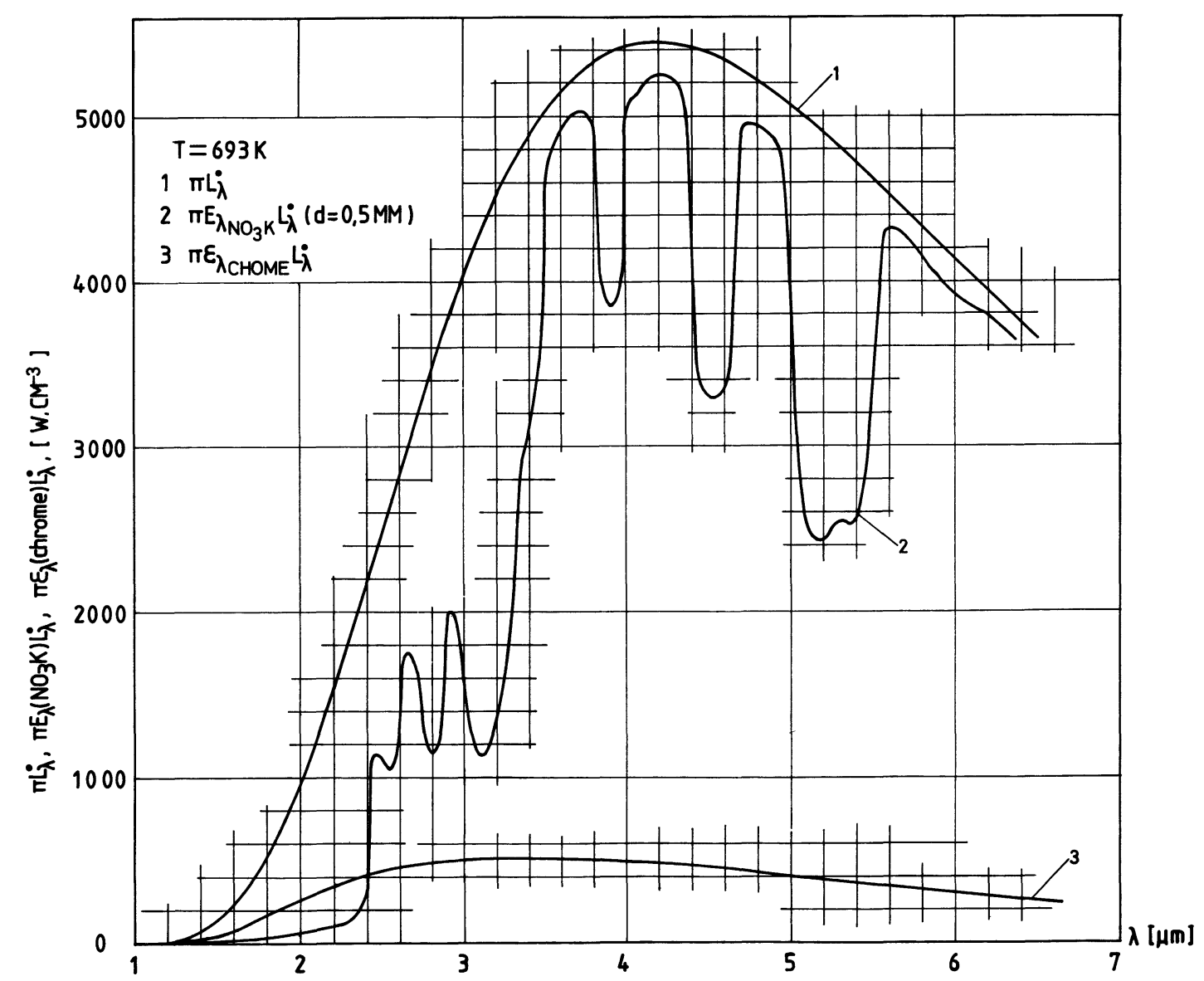

Fig. 6. - Répartition spectrale de l'émittance d'un corps noir à $693 \mathrm{~K}$, du produit $E_{\lambda} \pi L_{\lambda}^{0}$ pour une couche de $0,5 \mathrm{~mm}$ de $\mathrm{NO}_{3} \mathrm{~K}$ ainsi que du produit $\varepsilon_{\lambda \text { chrome }} \pi L_{\lambda}^{0}$.

[Spectral distribution of the emissive flux of a black body at $693 \mathrm{~K}$, of a $0.5 \mathrm{~mm}$ layer of $\mathrm{NO}_{3} \mathrm{~K}$ at $693 \mathrm{~K}$ and of the product $\varepsilon_{\lambda \text { chrome }} \pi L_{\lambda}^{0}$.] 
données expérimentales ou de la littérature. Le modèle de bande le plus simple consiste à décomposer le spectre réel en un ensemble de bandes rectangulaires de hauteur déterminée par le théorème de la moyenne. Dans notre cas le spectre de $\mathrm{B}_{2} \mathrm{O}_{3}$ est constitué de 11 bandes s'étendant entre 1 et $15 \mu \mathrm{m}$ (une bande de transparence totale, neuf bandes de semi-transparence, une bande d'opacité); pour les longueurs d'onde supérieures à $3,5 \mu \mathrm{m}$ les détails des bandes d'émission correspondant à des absorptions supérieures à $3000 \mathrm{~m}^{-1}$ ont été négligés. En ce qui concerne $\mathrm{NO}_{3} \mathrm{~K}$, le modèle spectral est composé de 16 bandes rectangulaires dont une de quasi-transparence, $10 \mathrm{de}$ semi-transparence et 5 d'opacité (voir Figs. 7a et $7 \mathrm{~b}$ ). Une modélisation analogue devrait pouvoir être faite pour l'indice de réfraction pour tenir compte de sa dispersion dans l'infrarouge. Pour $\mathrm{B}_{2} \mathrm{O}_{3}$, en l'absence d'information concernant l'indice dans l'infrarouge, celui-ci a été pris égal à 1,41 uniformément sur tout le spectre [16]. Dans le cas de $\mathrm{NO}_{3} \mathrm{~K}$, un découpage en bandes identique à celui du coefficient d'absorption a été effectué à partir de la formule (2).

L'émissivité des frontières par bande a été prise dans la littérature [18] et est représentée pour le cas typique du chrome sur la figure $7 \mathrm{~b}$.

b) L'analyse nodale consiste à décomposer le milieu en $N M$ tranches parallèles isothermes d'épaisseurs finies, le centre de chaque couche constituant un point nodal. On effectue un bilan énergétique local en chaque nœud $i$ (cf. appendice A) des échanges couplés conduction-rayonnement (en supposant dans notre cas connue la valeur de la conductivité thermique $K_{\mathrm{c}}$ ). Le bilan thermique en chaque nœud, à l'état stationnaire, conduit alors à un problème algébrique en termes de température nodale $\left(T_{i}\right)$. Sa solution numérique fournit le profil de température établi dans la couche pour les conditions aux limites imposées aux températures, un modèle d'absorption spectral et des émissivités de surface, donnés.

La densité de flux radiatif local au nœud $i$ à l'état stationnaire, échangée entre les surfaces $S_{1}$ et $S_{2}$, les

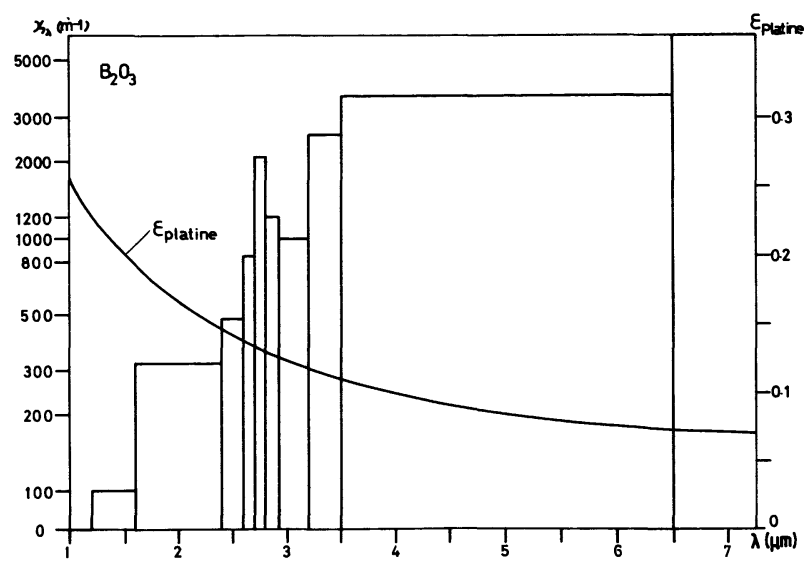

a)

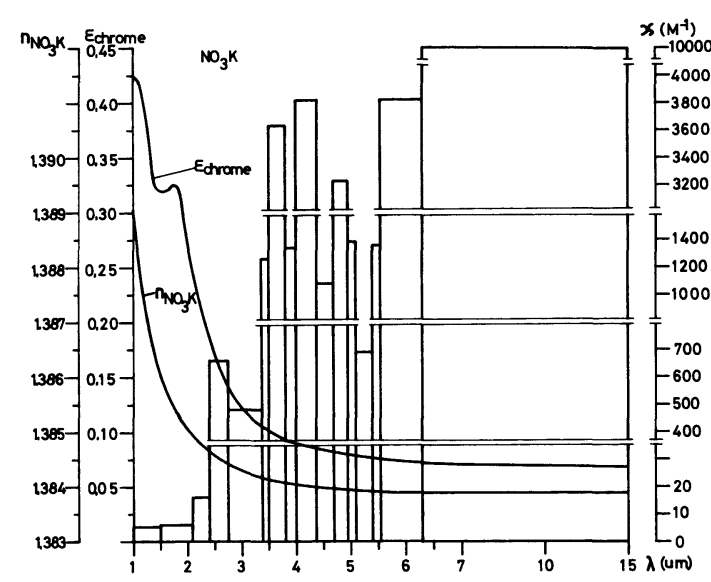

b)

Fig. 7. - Modèles de bandes rectangulaires du $\mathrm{B}_{2} \mathrm{O}_{3}(\mathrm{a})$ et du $\mathrm{NO}_{3} \mathrm{~K}(\mathrm{~b})$; émissivités spectrales du chrome (a), et du platine (b); dispersion de l'indice de réfraction du $\mathrm{NO}_{3} \mathrm{~K}(\mathrm{~b})$.

[Band models for $\mathrm{B}_{2} \mathrm{O}_{3}(\mathrm{a}), \mathrm{NO}_{3} \mathrm{~K}(\mathrm{~b})$; spectral emissivity of chromium (a), platinum (b); dispersion of refractive index $\mathrm{NO}_{3} \mathrm{~K}(\mathrm{~b})$.]

surfaces $S_{1}, S_{2}$ et les nœuds de volume, enfin entre les nœuds de volumes eux-mêmes s'obtient au moyen de l'expression :

$$
\begin{aligned}
q_{r}(i)= & \sigma_{\mathrm{B}} \sum_{k=1}^{N} n_{k}^{2}\left\{\left(\varepsilon_{1 k}\left(S_{1} S_{2}\right)_{k}\left(f_{k}\left(T_{S_{2}}\right) T_{S_{2}}^{4}-f_{k}\left(T_{S_{1}}\right) T_{S_{1}}^{4}\right)\right)+\sum_{j=1}^{i}\left(\varepsilon_{2 k}\left(S_{2} V_{j}\right)_{k}\left(f_{k}\left(T_{S_{2}}\right) T_{S_{2}}^{4}-f_{k}\left(T_{j}\right) T_{j}^{4}\right)\right)+\right. \\
& \left.+\sum_{j=i+1}^{N M}\left(\varepsilon_{1 k}\left(S_{1} V_{j}\right)_{k}\left(f_{k}\left(T_{j}\right) T_{j}^{4}-f_{k}\left(T_{S_{1}}\right) T_{S_{1}}^{4}\right)\right)+\sum_{j=i+1}^{N M} \sum_{l=1}^{i}\left(\left(V_{j} V_{l}\right)_{k}\left(f_{k}\left(T_{j}\right) T_{j}^{4}-f_{k}\left(T_{l}\right) T_{l}^{4}\right)\right)\right\}
\end{aligned}
$$

où pour chaque bande $k(k=1, \ldots, N): n_{k}$ est l'indice de réfraction, $f_{k}\left(T_{j}\right)$ est la fraction spectrale émise par la bande pour la température $T_{j}$ du nœud $j(j=1, \ldots, N M), \varepsilon_{i k}$ l'émissivité spectrale de la surface $S_{i} \sigma_{\text {B }}$ est la constante de Stefan-Boltzmann. Les nota- tions symboliques $\left(S_{1} S_{2}\right)_{k},\left(S_{1} V_{j}\right)_{k},\left(V_{i} V_{j}\right)_{k}$ utilisées par Hottel et Sarofim [21] sont associées au phénomène de multiréflexions, elles désignent les aires d'échanges étendus, respectivement entre deux surfaces, une surface et un volume, ainsi que deux volumes isothermes. 
Elles s'introduisent dans le calcul des flux nets lorsqu'on écrit que celui-ci est proportionnel au pouvoir émissif de chaque nœud dans une bande spectrale donnée. Ces grandeurs (de la dimension d'une surface) dépendent de l'émissivité des surfaces extérieures et des aires d'échanges directs $\left(s_{1} s_{2}\right)_{k}$ calculées pour des surfaces noires. On trouvera les expressions détaillées de $\left(S_{1} S_{2}\right)_{k}$, $\left(S_{1} V_{j}\right)_{k}$ et $\left(V_{i} V_{j}\right)_{k}$ ainsi que leur dérivation en fonction des aires d'échanges directs $\left(s_{1} s_{2}\right)_{k}$ de surfaces noires, au moyen des relations de Gebhart dans [3].

Le premier terme entre crochets de (3) représente relativement au nœud $i$, les contributions radiatives totales dues aux échanges via les multiréflexions entre les surfaces $S_{1}$ et $S_{2}$, les second et troisième termes correspondent aux flux traversant le nœud $i$ échangés entre les surfaces frontières et les nœuds de volume, le quatrième aux échanges entre les nœuds de volume situés de part et d'autre du nœud $i$ considéré.

L'interaction entre le rayonnement et la conduction entraîne une déformation des profils de température comme le montre la figure A.1 de l'appendice A, calculée pour une couche de $\mathrm{B}_{2} \mathrm{O}_{3}$ et des frontières faiblement émissives aux températures de $1250 / 1350 \mathrm{~K}$. La distribution de flux local correspondant est représentée sur la figure 8. De grandes variations locales peuvent être observées, notamment aux frontières où la participation conductive augmente fortement. En conséquence, une évaluation commode de la contribution radiative, associée au mode conductif, dans un transfert couplé peut être tentée en calculant le flux radiatif moyenné sur l'épaisseur de la couche, à l'aide d'une formule de quadrature appliquée aux flux radiatifs locaux. Dans ces conditons, on pourra écrire la relation phénoménologique entre le flux moyen calculé et le gradient thermique extérieur appliqué $\Delta T / d$

$$
\bar{q}_{r}=-K_{r} \Delta T / d
$$

Elle introduit un coefficient effectif de conductivité thermique $K_{\mathrm{r}}$ d'origine radiative, effectif, car dépendant des conditions imposées au système et non des propriétés intrinsèques du matériau.

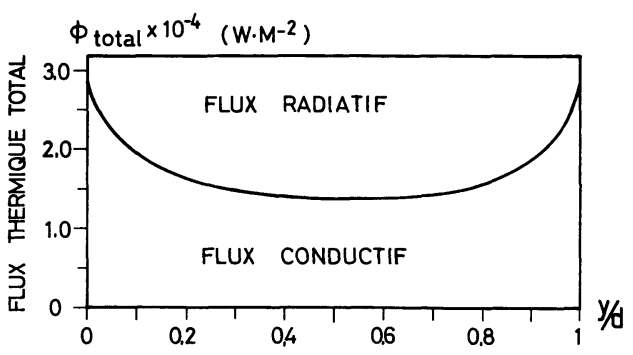

Fig. 8. - Distribution calculée des flux pour une couche de $\mathrm{B}_{2} \mathrm{O}_{3}$ de $10 \mathrm{~mm}$.

[Calculated flux distribution within a $10 \mathrm{~mm}$ slab of $\mathrm{B}_{2} \mathrm{O}_{3}$.]
4.2 RÉSultats DE LA MODÉlisation. - L'évolution de $K_{\mathrm{r}}$ en fonction de l'épaisseur du mur est représentée sur la figure 9 dans le cas de $\mathrm{NO}_{3} \mathrm{~K}$. La valeur de la conductivité thermique de ce sel a été prise dans la référence [8] $\left(0,42 \mathrm{~W} \cdot \mathrm{m}^{-1} \mathrm{~K}^{-1}\right.$ entre 620 et $720 \mathrm{~K})$. Le flux réduit $q_{\mathrm{r}} /(\Delta T / d)$ a été calculé pour différentes valeurs de l'émissivité des surfaces, entre les températures de 600 et $720 \mathrm{~K}$, pour le cas de surfaces présentant une différence de température de $10 \mathrm{~K}$, ce qui correspond aux conditions expérimentales que l'on trouve dans [78].

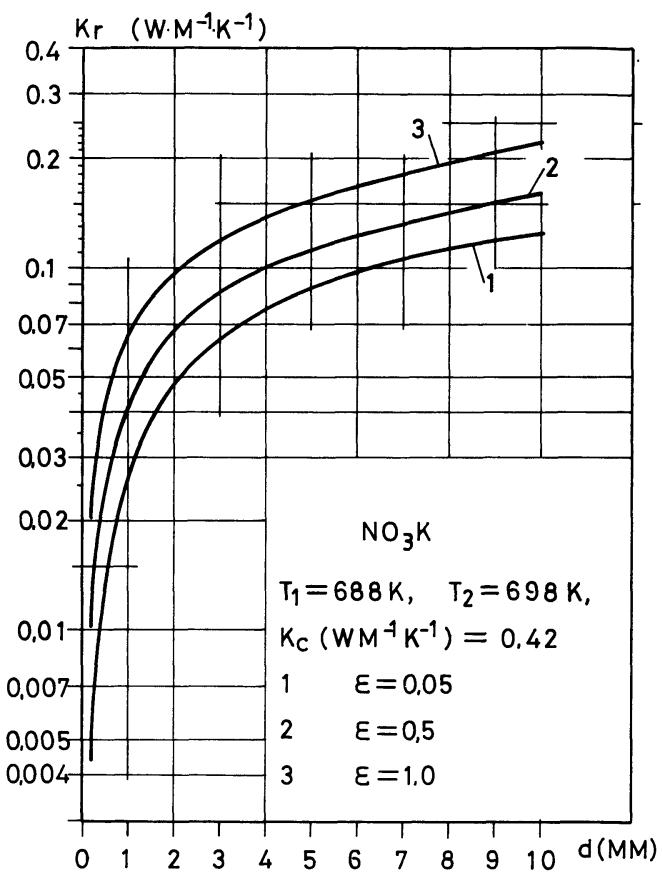

Fig. 9. - Variation de la conductivité radiative de $\mathrm{NO}_{3} \mathrm{~K}$ fondu en fonction de l'épaisseur, pour diverses valeurs de l'émissivité des parois à $693 \mathrm{~K}$.

[Radiative conductivity of molten $\mathrm{NO}_{3} \mathrm{~K}$, versus thickness, for various walls emissivities at $693 \mathrm{~K}$.]

On note pour les parois grises de faible émissivité, une contribution radiative, relativement à la partie conductive, inférieure au \% pour les épaisseurs plus petites que $0,2 \mathrm{~mm}$ et de l'ordre de $27 \%$ pour une couche d'épaisseur $10 \mathrm{~mm}$. Pour les émissivités élevées $\left(\varepsilon_{\lambda}=1\right)$ la participation du rayonnement est intense mais le rapport $K_{\mathrm{r}} / K_{\mathrm{c}}$ est pratiquement le même pour la configuration $(d=10 \mathrm{~mm}, \varepsilon=1)$ que l'écart de température entre les surfaces soit de $10 \mathrm{~K}$ ou de $100 \mathrm{~K}$; pour cette configuration $(d=10 \mathrm{~mm}, \varepsilon=1)$, la contribution radiative calculée atteint $52 \%$ de la valeur du cas purement conductif.

Le spectre de la densité relative du flux radiatif par unité de longueur d'onde $\left(q_{\mathrm{r} k}(j) / \Delta \lambda_{k}\right) / \Sigma q_{\mathrm{r} k}(j)$ mesure l'importance respective des bandes spectrales dans le transfert local ; pour $\mathrm{NO}_{3} \mathrm{~K}$ il est illustré sur la figure 10 . 


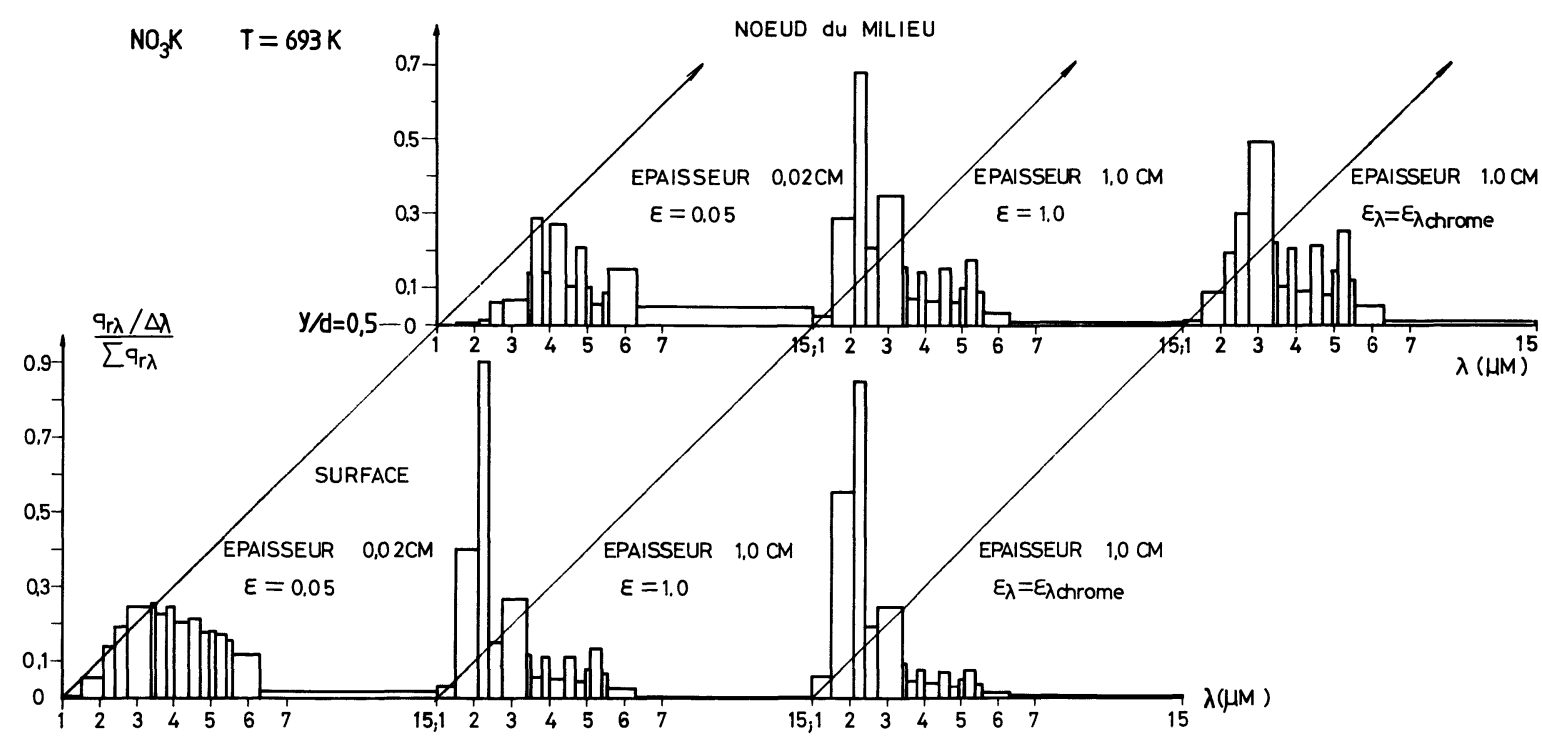

Fig. 10. - Spectre de la densité de flux radiatif de diverses épaisseurs de couches de $\mathrm{NO}_{3} \mathrm{~K}$ et diverses émissivités de surface, en fonction de la distance à la frontière.

[Spectra of the radiative flux density of various $\mathrm{NO}_{3} \mathrm{~K}$ layers thicknesses with different surface's emissivities as a function of distance.]

On peut faire les constatations suivantes :

- en surface dans le cas d'une lame mince de $0,2 \mathrm{~mm}$ d'épaisseur et d'émissivité uniforme, égale à 0,05 (correspondant aux conditions expérimentales de [8]), $82 \%$ de la contribution radiative provient des bandes situées entre 1 et $6,3 \mu \mathrm{m}$, dont $30 \%$ pour la seule région $1-3,4 \mu \mathrm{m}$; pour le système épais à forte émissivité $(d=10 \mathrm{~mm}, \varepsilon=1), 95 \%$ de l'énergie est émis dans la bande 1-6,3 $\mu \mathrm{m}$ dont $75 \%$ par la seule bande $1-2,4 \mu \mathrm{m}$; on peut apprécier l'augmentation du rôle de la fenêtre de transparence lorsque les frontières deviennent très émissives (Fig. 10a, b) ;

- au centre du mur, la répartition spectrale du flux est considérablement modifiée pour le cas de l'échantillon mince et d'émissivité faible, comme le montre la figure $10 \mathrm{~b}$ pour le système $(d=0,2 \mathrm{~mm}, \varepsilon=0,05)$; c'est maintenant la région $6,3-15 \mu \mathrm{m}$ qui contribue pour $44 \%$ de l'énergie radiative locale contre seulement $7 \%$ à la région 1-3,4 $\mu \mathrm{m}$; l'émission des surfaces étant atténuée, on retrouve un spectre très proche de celui du fluide représenté sur la figure 6. Pour un échantillon de forte épaisseur et d'émissivité élevée l'influence des surfaces continue à se faire sentir (Fig. 10d) et on observe peu de modifications par raport au flux propre en surface ; $94 \%$ de l'énergie reste émise dans l'intervalle $1-3,6 \mu \mathrm{m}$ dont $69 \%$ dans celui $1-3,4 \mu \mathrm{m}$. Pour le cas de l'épaisseur $10 \mathrm{~mm}$ et de frontière en chrome on se retrouve dans une situation intermédiaire (Fig. 10f).

Sur la figure 11 a été reportée l'évolution avec l'épaisseur du rapport $K_{\mathrm{r}} /\left(K_{\mathrm{r}}+K_{\mathrm{c}}\right)$ d'une couche de $\mathrm{B}_{2} \mathrm{O}_{3}$ pour différentes émissivités de parois, ainsi que pour différentes températures allant de 700 à $1200 \mathrm{~K}$ lorsque le gradient thermique est constant $(100 \mathrm{~K} / \mathrm{cm})$.

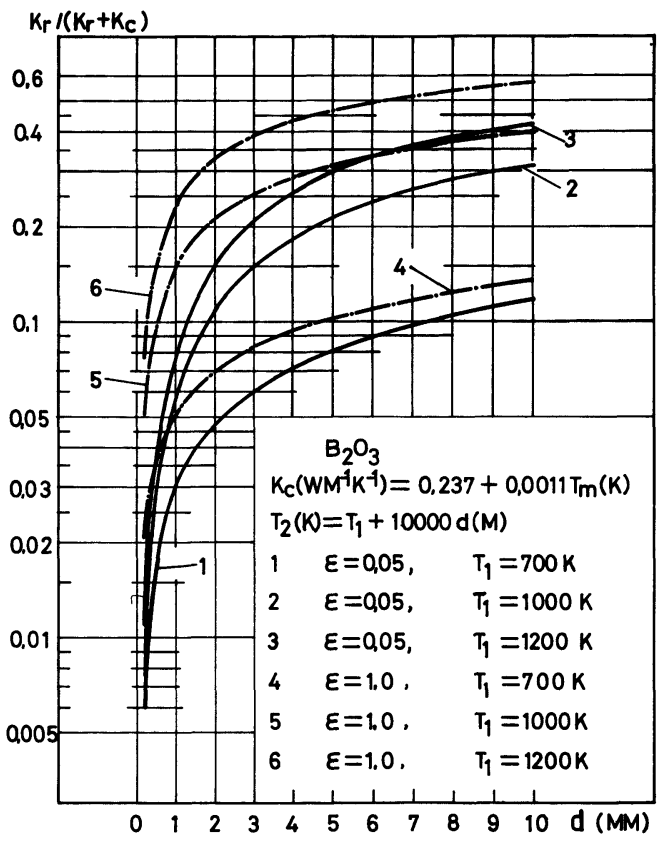

Fig. 11. - Variation de la conductivité radiative réduite avec l'épaisseur de $\mathrm{B}_{2} \mathrm{O}_{3}$ pour diverses valeurs de la température et d'émissivité de surfaces considérées comme grises.

[Reduced radiative conductivity of $\mathrm{B}_{2} \mathrm{O}_{3}$ against thickness for various temperature and gray surfaces emissivities.]

On relève que pour une lame mince $(1 \mathrm{~mm})$ d'émissivité grise faible $(\varepsilon=0,05)$ à la température de $1200 \mathrm{~K}$, $7 \%$ seulement de l'énergie est échangée par voie radiative contre près de $45 \%$ dans une lame épaisse $(10 \mathrm{~mm})$; pour cette même température, avec des systèmes d'épaisseurs élevées mais d'émissivités fortes, 
le couplage est très actif puisque la contribution radiative représente $57 \%$ du transfert total.

4.3 Comparaison avec le modèle de Poltz. Poltz et Jugel $[1,22]$ ont proposé une expression de la densité de flux radiatif participant au transfert dans un milieu solide transparent à frontières non grises de dimensions finies. Leur modèle est construit sur la base des hypothèses suivantes :

- le profil de température est supposé linéaire et le gradient de température appliqué faible,

- on peut développer la fonction de Planck $L_{\nu}^{0}(T(x))$ en série de Taylor.

En calculant la moyenne de la densité de flux sur la profondeur optique $\tau_{0 k}=\kappa_{k} d$, le modèle de Poltz aboutit dans le cas d'une représentation du spectre d'absorption par un ensemble de bandes rectangulaires à l'expression :

$$
K_{\mathrm{r}}\left(d, \varepsilon_{k}\right)=\frac{4 \pi}{3} \sum_{k=1}^{N} \frac{n^{2}}{\kappa_{k}} \int \frac{\partial L_{\nu}}{\partial T} Y\left(\varepsilon_{k}, \tau_{0 k}\right) \mathrm{d} \nu
$$

où $Y\left(\varepsilon_{k}, \tau_{0 k}\right)$ est un coefficient numérique dépendant des conditions radiatives aux frontières et de l'épaisseur optique $\tau_{0 k}$ qui s'écrit lorsque les émissivités $\varepsilon_{k}$ des frontières sont identiques :

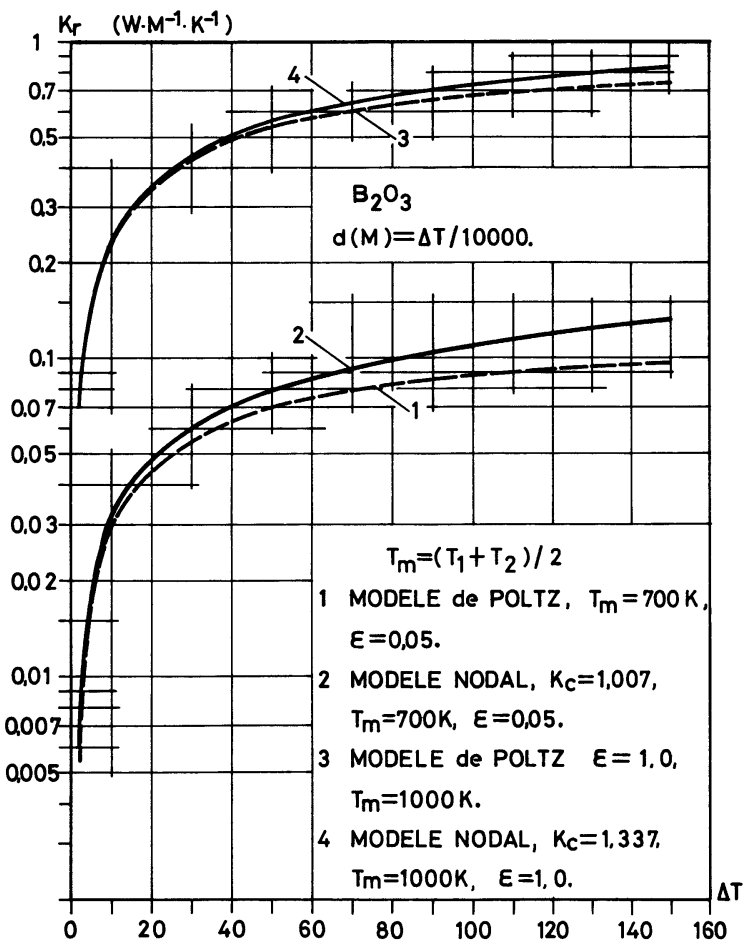

Fig. 12. - Evolution de la conductivité radiative avec l'écart de température aux frontières de $\mathrm{B}_{2} \mathrm{O}_{3}$. Comparaison avec le modèle de Poltz.

[Evolution of the radiative conductivity versus temperature difference of the boundaries in $\mathrm{B}_{2} \mathrm{O}_{3}$. Comparison with Poltz's model.]

$$
\begin{aligned}
Y\left(\varepsilon_{k}, \tau_{0 k}\right)=( & \left.-\frac{3}{4 \tau_{0 k}}\left(1-4 E_{5}\left(\tau_{0 k}\right)\right)\right)- \\
& -\frac{2}{3 \tau_{0}} \frac{1-\varepsilon_{k}-2\left(1-\varepsilon_{k}\right)^{2} E_{3}\left(\tau_{0 k}\right)}{1-4\left(1-\varepsilon_{k}\right)^{2} E_{3}^{2}\left(\tau_{0 k}\right)} \times \\
& \times\left(1-3 E_{4}\left(\tau_{0 k}\right)\right)^{2}
\end{aligned}
$$

où les $E_{n}\left(\tau_{0 k}\right)$ désignent les fonctions exponentielles intégrales d'ordre $n$.

Les résultats de la modélisation par l'analyse nodale et ceux provenant du modèle de Poltz ont été comparés sur les figures 12 et 13 dans le cas d'une couche de $\mathrm{B}_{2} \mathrm{O}_{3}$. Sur la figure 12 l'évolution de $K_{\mathrm{r}}$ est représentée en fonction de l'écart de température imposé à la couche pour deux températures différentes $(700$ et $1000 \mathrm{~K}$ et ce pour des valeurs $K_{\mathrm{c}}$ correspondant à ces températures suivant la référence [9]; la figure 13 montre la variation de $K_{\mathrm{r}}$ avec l'épaisseur pour une couche de $\mathrm{NO}_{3} \mathrm{~K}$. On observe un bon accord général entre les résultats de l'analyse nodale et du modèle de Poltz pour les émissivités de surface élevées, un peu moins bon pour les émissivités faibles. Un écart important apparaît cependant lorsque les différences de température entre les surfaces sont fortes, conditions qui ne correspondent plus aux hypothèses qui ont servi à établir le modèle de Poltz.

\section{Discussion.}

A l'état stationnaire en tout point du milieu, si $q_{\mathrm{t}}, q_{\mathrm{c}}$ et $q_{\mathrm{r}}$ sont respectivement les composantes selon l'axe $x$ des densités de flux total, conductif et radiatif, en négligeant le transfert convectif, on peut écrire :

$$
q_{\mathrm{t}}=q_{\mathrm{c}}(x)+q_{\mathrm{r}}(x) .
$$

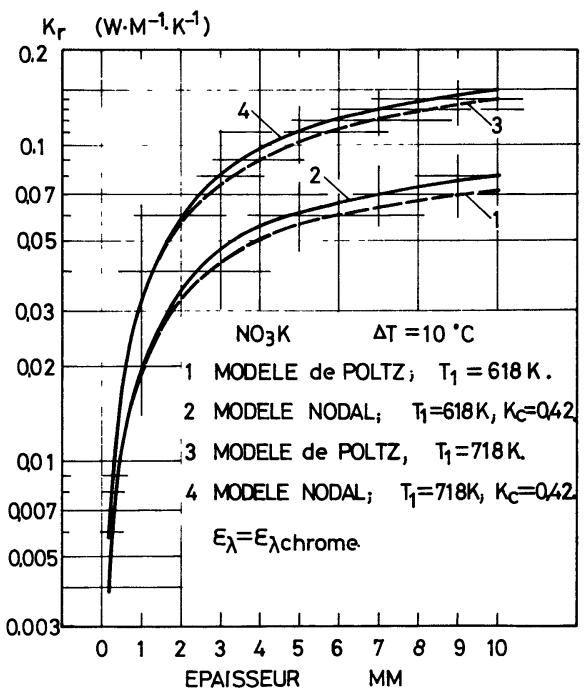

Fig. 13. - Evolution de la conductivité radiative en fonction de l'épaisseur de la couche de $\mathrm{NO}_{3} \mathrm{~K}$ fondu. Comparaison avec le modèle de Poltz.

[Evolution of the radiative conductivity in molten $\mathrm{NO}_{3} \mathrm{~K}$ against thickness. Comparison with Poltz's model.] 
Dans les expériences de cylindres coaxiaux ou de couche plane, $q_{\mathrm{t}}$ est la densité de flux de chaleur mesurée (émise par l'élément chauffant). Les profils de température sont déformés par le couplage, ce qui est le cas pour les gradients considérés au $\S$ précédent, et séparément, les flux $q_{\mathrm{c}}(x)$ et $q_{\mathrm{r}}(x)$ varient localement, on peut cependant calculer un flux radiatif moyen $\bar{q}_{\mathrm{r}}$; une approche de la conductivité thermique d'origine moléculaire ou phonique sera donnée alors, par l'expression :

$$
K_{\mathrm{c}}=\left(q_{\mathrm{t}}-\bar{q}_{\mathrm{r}}\right) /(\Delta T / d)
$$

Si les données publiées n'ont pas été l'objet d'une correction radiative préalable on aura :

$$
K_{\text {c vraie }}=K_{\text {clue }}-K_{\mathrm{r}}(d, \varepsilon, \Delta T) .
$$

En possession des résultats du $\S 4$, et lorsque les conditions expérimentales des données publiées (écart de température, distance de séparation et nature des parois) sont connues - ce qui n'est pas toujours le cas - il devient possible de proposer des valeurs numériques pour la part revenant au transfert radiatif dans les mesures de conductivité thermique des sels fondus et des fontes vitreuses.

Dans ce qui suit, pour le nitrate de potassium fondu, nous limiterons la discussion de la correction radiative aux résultats de White et Davis [23], Mc Donald et Davis [24], ainsi que de ceux, plus récents, de Santini et al. et de Tufeu et al.

Les mesures de White et Davis et de Mc Donald et Davis ont été obtenues au moyen de conductimètres à cylindres coaxiaux en argent poli verticaux gardés, d'épaisseur respectivement de 3,18 et $2,54 \mathrm{~mm}$. Dans les expériences de Tufeu et al. l'appareillage est identique mais l'espacement des cylindres a été réduit à $0,2 \mathrm{~mm}$ et l'écart de température entre les parois est d'environ 1,5 K.

Les mesures de Santini et al. ont été effectuées dans un conductimètre horizontal à plaques planes gardées espacées de $9 \mathrm{~mm}$, les frontières sont formées de plaques de cuivre protégées par un chromage et la différence de température imposée est de $10 \mathrm{~K}$.

Dans le cas de $\mathrm{B}_{2} \mathrm{O}_{3}$, nos commentaires porteront sur les mesures de conductivité thermique effectuées jusqu'à $1270 \mathrm{~K}$ par G. K. Creffield et A. J. Wickens [9] à l'aide d'une méthode de cylindres coaxiaux construits en platine, celles de Lezhenin et al. [10] ainsi que celles de Nishimura et al. [25].

5.1 CAS DES SURFACES GRISES. - La correction radiative à apporter aux données de conductivité thermique de $\mathrm{NO}_{3} \mathrm{~K}$ fondu peut être déterminée à partir des courbes de la figure 9 . On voit qu'à $700 \mathrm{~K}$, dans les conditions expérimentales de Santini et al., la contribution radiative est de $26 \%$ par rapport à la conductivité thermique si l'émissivité des frontières est considérée comme grise et prise égale à 0,05 ; elle passe à $31 \%$ si l'émissivité est de 0,1. Pour le dispositif de Tufeu et al., la participation du rayonnement $\left(\varepsilon_{\text {argent }}=0,03\right.$ entre 1 et $15 \mu \mathrm{m})$ est inférieure à $1 \%$.

Lorsque l'on prend pour base les données de Tufeu ainsi corrigées, la conductivité thermique attendue dans les expériences de Santini devraient atteindre, à $700 \mathrm{~K}$, la valeur de $0,54 \mathrm{~W} \cdot \mathrm{m}^{-1} \mathrm{~K}^{-1}$ alors que la valeur annoncée est de $0,47 \pm 0,04$; la correction radiative que nous proposons ne permet pas de trouver un accord entre ces deux séries de mesure (voir Fig. 14). En revanche, en procédant de la même manière avec les données de White et Davis et de Mc Donald et Davis, les valeurs attendues sont de $0,49 \mathrm{~W} \cdot \mathrm{m}^{-1} \mathrm{~K}^{-1}$ en bon accord avec celle annoncée par ses auteurs de $0,48 \mathrm{~W} \cdot \mathrm{m}^{-1} \mathrm{~K}^{-1}$ et de

\begin{tabular}{|c|c|c|}
\hline$K_{\exp }$ & $K_{\text {eff }}$ (MODELE NODAL, $K_{c}=0.42$ ) & $K_{c}=K_{\exp }-K_{r}$ \\
\hline - Mc Donald I22I & $1 \mathrm{~d}=9 \mathrm{MM}, \varepsilon_{\lambda \text { ctrome. }}$. & $5 K_{\exp } / 221-K_{r}$ \\
\hline + White $121 I$ & $2 \mathrm{~d}=3 \mathrm{MM}, \quad E_{\lambda c h r o m e .}$. & $6 \mathrm{~K} \exp / 21 /-K_{r}$ \\
\hline$\times$ Santini 171 & $3 \mathrm{~d}=2.54 \mathrm{MM}_{i} \varepsilon_{1 \lambda \text { argent }}, \varepsilon_{2 \lambda \text { acier inoxydable }}$ & $7 K_{\exp } / 81-K_{r}$ \\
\hline - Tufeu 181 & $4 \mathrm{~d}=0.2 \mathrm{MM} \quad \varepsilon_{\text {גargent }}$ & \\
\hline
\end{tabular}
$0,50 \mathrm{~W} \cdot \mathrm{m}^{-1} \mathrm{~K}^{-1}$ respectivement.

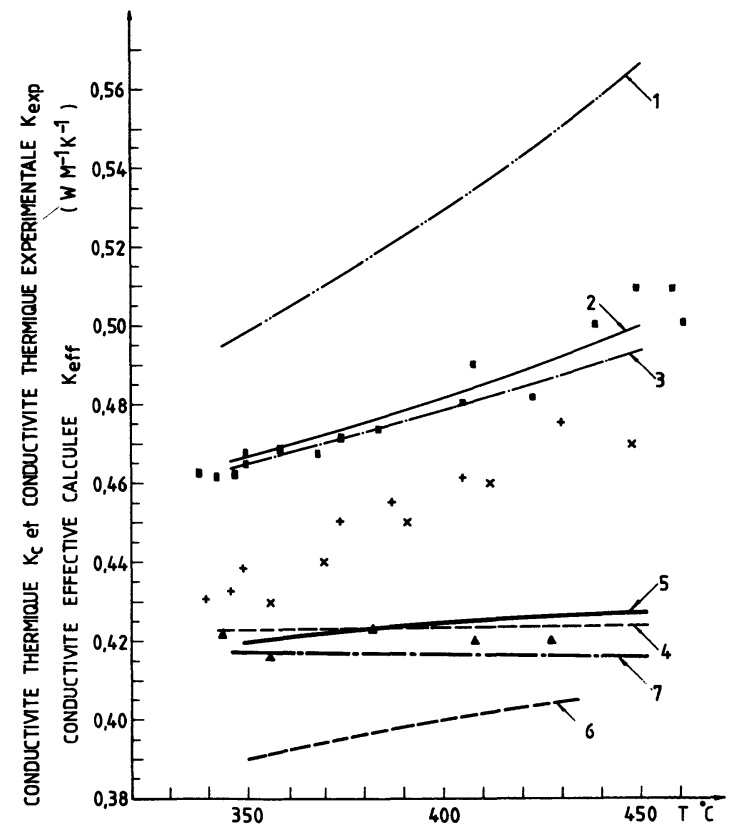

Fig. 14. - Evolution de la conductivité thermique de $\mathrm{NO}_{3} \mathrm{~K}$ avec la température selon plusieurs auteurs et correction radiative.

[Evolution of the thermal conductivity of molten $\mathrm{NO}_{3} \mathrm{~K}$ versus temperature following several authors and radiative correction.]

Remarquons que la similitude des spectres infrarouges de la famille des nitrates alcalins laisse prévoir pour ces sels une contribution radiative au transfert couplé du même ordre de grandeur que pour le cas du nitrate de potassium. 
La compilation des données de la conductivité thermique de $\mathrm{B}_{2} \mathrm{O}_{3}$ montre que dès $600 \mathrm{~K}$ il existe un désaccord sérieux entre les mesures qui atteint un facteur six à $1300 \mathrm{~K}$. En nous référant aux résultats de la figure 11 , nous relevons que, pour des mesures faites à l'aide de systèmes à frontières grises peu émissives $(\varepsilon=0,05)$, l'expérimentation dans une couche de $5 \mathrm{~mm}$ d'épaisseur entraîne une contribution radiative calculée sur la base des données de la référence [9], de $43 \%$ à $1250 \mathrm{~K}$, c'est-à-dire de l'ordre des écarts constatés entre les résultats des références [9, 10, 25].

Les résultats expérimentaux de Creffield et Wickens entre 570 et $1270 \mathrm{~K}$ ont été obtenus par la méthode des cylindres coaxiaux verticaux pour des épaisseurs de matières comprises entre 2 et $5 \mathrm{~mm}$, puis extrapolés pour une épaisseur nulle. Il paraissait intéressant de tester leur méthodologie à partir de la modélisation des transferts radiatifs dans les conditions de fonctionnement du thermo-conductimètre employé par ces auteurs. Nous avons reporté sur la figure 15, pour la température de $1250 \mathrm{~K}$, la variation avec l'épaisseur de la conductivité globale $\left(K_{\mathrm{r}}+K_{\mathrm{c}}\right)$ de différentes couches de $\mathrm{B}_{2} \mathrm{O}_{3}$; on note que l'extrapolation pour une épaisseur nulle conduit bien à la valeur fournie par ces auteurs. Dans ces conditions, le signe positif de l'effet de température sur la conductivité thermique de $\mathrm{B}_{2} \mathrm{O}_{3}$ à l'état de fonte peut être considéré comme assuré.

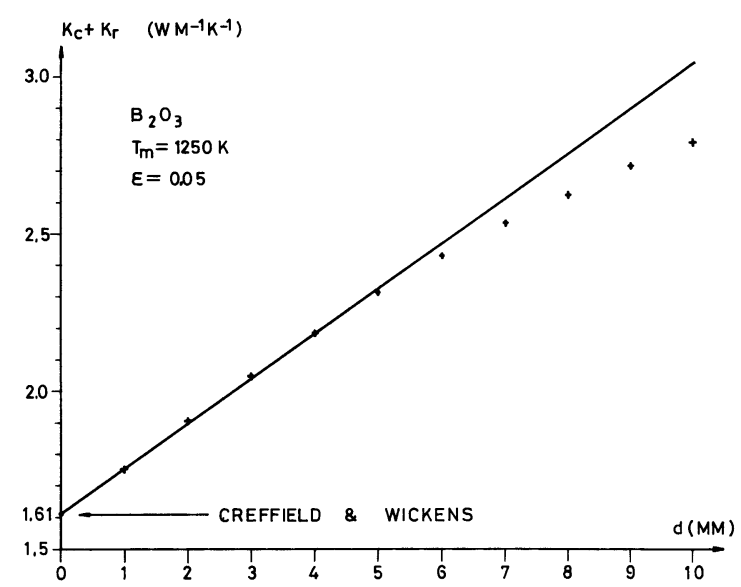

Fig. 15. - Extrapolation à épaisseur nulle de l'évolution de $K_{\mathrm{r}}+K_{\mathrm{c}}$ en fonction de l'épaisseur de la couche; les points représentent les résultats de calcul par l'analyse nodale.

[Extrapolation to nul thickness of the $K_{\mathrm{r}}+K_{\mathrm{c}}$ evolution as a function of the slab thickness; full dots are nodal analysis results.]

5.2 CAS DES SURFACES NON GRISES. - On sait que, si, dans le proche infrarouge, des métaux, comme l'argent ou l'aluminium, polis ont des émissivités pratiquement indépendantes de la longueur d'onde, il n'en est pas de même pour un grand nombre d'autres métaux, tels que le platine et le chrome, métaux pouvant entrer dans la conception des cellules de conductivité thermique capables de fonctionner à très haute température en milieux agressifs. Dans le cas des métaux cités (voir Figs. 7a et 7b), l'émissivité est importante dans l'infrarouge proche jusqu'à $6 \mu \mathrm{m}$; ainsi pour le chrome elle est comprise entre 0,40 et 0,30 dans l'intervalle 1 à $2,5 \mu \mathrm{m}$, comprise entre 0,3 et 0,2 dans l'intervalle 2,5 et $4 \mu \mathrm{m}$; elle peut être considérée constante et égale à 0,07 à partir de $5 \mu \mathrm{m}$.

Les résultats de la modélisation, tenant compte de la variation spectrale de l'émissivité des parois, montre dans ces conditions un certain accroissement de la contribution radiative à haute température.

Pour le nitrate de potassium contenu entre des parois chromées, la participation radiative au transfert dans une couche de $9 \mathrm{~mm}$ d'épaisseur s'élève à $30 \%$ à $693 \mathrm{~K}$. Le spectre de la densité relative de flux radiatif par bande pour cette dernière température est représenté sur les figures $10 \mathrm{e}$ et $10 \mathrm{f}$. On y observe, pour une couche épaisse, l'importance des bandes d'émission aux parois dans l'infrarouge proche et leur régression à l'intérieur du matériau dans lequel le transfert est dominé par le spectre propre de la substance. Ceci met en évidence la transformation spectrale de la composante radiative de l'énergie sous l'effet du couplage avec la conduction à mesure que l'on progresse dans les couches internes.

On a représenté sur la figure 16 l'évolution de la contribution radiative au transfert d'une fonte de $\mathrm{B}_{2} \mathrm{O}_{3}$ de $10 \mathrm{~mm}$ d'épaisseur entre 700 et $1250 \mathrm{~K}$. En l'absence d'indications précises des auteurs sur les conditions expérimentales mises en œuvre dans leurs mesures de conductivité thermique de $\mathrm{B}_{2} \mathrm{O}_{3}$, les calculs

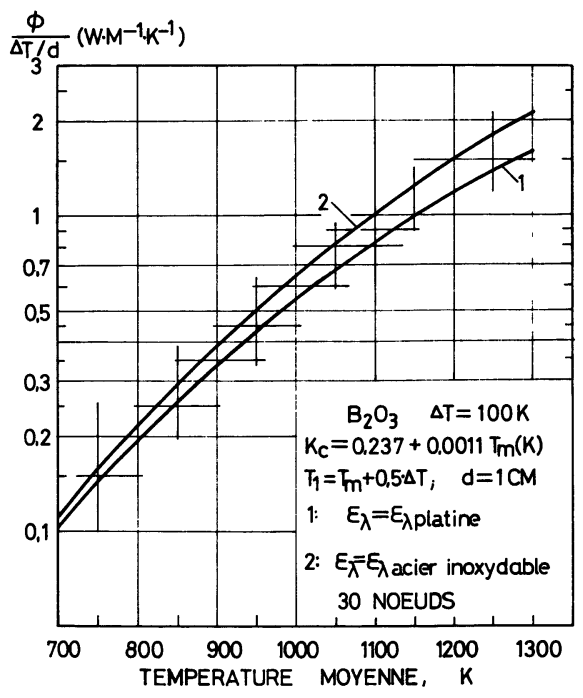

Fig. 16. - Variation avec la température du flux radiatif réduit d'une couche d'épaisseur de $\mathrm{B}_{2} \mathrm{O}_{3}$ pour des surfaces 1) de platine et 2) d'acier inoxydable altéré.

[Variation against temperature of the mean reduced radiative flux of a $10 \mathrm{~mm}$ thickness of $\mathrm{B}_{2} \mathrm{O}_{3}$ fitted with 1) platinum surfaces, 2) altered stainless steel surfaces.] 
ont été effectués en supposant les frontières 1) en platine, 2) en acier inoxydable altéré par un chauffage prolongé en milieu agressif. On constate qu'à $1250 \mathrm{~K}$, par référence aux données de Creffield et Wickens, que pour des surfaces de platine, la part de la conductivité radiative représente maintenant $87 \%$ de celle de la conductivité moléculaire, elle passe à $112 \%$ pour des surfaces en acier inoxydable.

\section{Conclusion.}

Les résultats de la modélisation du transfert radiatif dans les milieux semi-transparents à l'état fondu, obtenus à partir de la résolution numérique des équations (1D) du transfert couplé rayonnement-conduction, ont montré la nécessité de prendre en compte des spectres d'absorption détaillés des substances c'est-àdire de représenter par un spectre multi-bandes les propriétés d'absorption, les variations spectrales de l'émissivité des surfaces frontières ainsi que celles de l'indice de réfraction, notamment pour les températures supérieures à $1000 \mathrm{~K}$.

Ils ont permis de calculer la correction radiative à apporter dans les mesures de conductivité thermique des sels fondus, effectuées en permanent, lorsqu'une méthode d'identification n'a pu être mise en cuvre pour déterminer cette grandeur et la dégager des effets du couplage conduction-rayonnement. On a pu voir que le souci majeur des expérimentateurs, qui a été longtemps celui de minimiser les erreurs introduites par les effèts convectifs, ne s'était pas toujours accompagné (du moins en régime stationnaire) du même effort visà-vis des effets de rayonnement, trop légèrement sousestimés. Il a été constaté que la grande confusion relativement au signe de l'effet de la température sur la conductivité thermique des nitrates alcalins en est une conséquence.

L'application de la modélisation des transferts radiatifs a conduit, dans le cas du nitrate de potassium fondu, à admette une insensibilité de cette grandeur par rapport à la température; en revanche le signe positif obtenu par les expérimentateurs pour l'anhydride borique semble confirmé.

Enfin il a été possible de montrer, qu'à moindres frais de calcul, l'approximation de Poltz, considérée dans le cas non gris, fournit pour la correction radiative à la conductivité thermique, des résultats satisfaisants lorsqu'elle se trouve être appliquée aux conditions de mesure les plus courantes.

\section{Appendice A.}

Le calcul séparé des composantes radiatives et conductives lors d'un transfert combiné dans un milieu semitransparent passe par la connaissance du champ des températures établi dans le système. Dans le cas stationnaire, celui-ci est solution de l'équation :

$$
\operatorname{div}\left(\mathbf{q}_{\mathrm{c}}+\mathbf{q}_{\mathrm{r}}\right)=0
$$

où $\mathbf{q}_{\mathrm{c}}$ est la densité de flux conductif, dépendant du REVUE DE PHYSIQUE APPLIQUÉE. -T. 22, N’2, FÉVRIER 1987 gradient local de température par la loi de Fourier, et $\mathbf{q}_{\mathrm{r}}$ la densité de flux radiatif, donnée par l'expression (3) du texte. C'est une fonctionnelle dépendant du champ des températures ainsi que des conditions aux limites radiatives.

Le problème du mur non gris à températures imposées aux parois, n'a donc en général de solution que par voie numérique et c'est par l'analyse nodale que les calculs ont été présentés.

Lorsque la loi de Fourier est insérée dans (A.1), pour le cas du problème à une dimension, après intégration de cette équation sur l'axe des $x$ entre les abscisses $i-1 / 2$ et $i+1 / 2$, puis application du théorème de Green à l'intégrale contenant le flux radiatif, l'équation (A.l) prend la forme discrète suivante :

$$
-\frac{K_{\mathrm{c}}}{\Delta X}\left(T_{i+1}+T_{i-1}-2 T_{i}\right)+\phi_{\mathrm{r}}(i)=0
$$

où les $T_{i}$ désignent la température au nœud $i$, $\Delta X$ étant la distance internodale, $K_{\mathrm{c}}$ est la conductivité thermique du milieu et $\phi_{\mathrm{r}}(i)$ exprime le bilan radiatif au nœud $i$.

Le problème de la détermination du champ de température pour le transfert couplé est donc ramené à celui du mur plan conductif avec une source $\phi_{\mathrm{r}}(i)$ non linéaire.

Si $q_{\mathrm{r}}(i), q_{\mathrm{r}}(i-1)$ sont les densités de flux radiatifs totaux, respectivement aux nœuds $i$ et $i-1$, pour un système plan semi-transparent bordé par des frontières $S_{1}$ et $S_{2}$ d'émissivité $\varepsilon_{1}$ et $\varepsilon_{2}$, le terme de source s'écrit d'après l'expression (3) du texte :

$$
\begin{aligned}
\phi_{\mathrm{r}}(i)=g_{\mathrm{r}}(i) & -q_{\mathrm{r}}(i-1)= \\
=\sigma \sum_{k=1}^{N} & n_{k}^{2}\left\{\sum_{j=1}^{N M}\left(V_{i} V_{j}\right)_{k}\left(a_{k T_{j}} T_{j}^{4}-a_{k T_{i}} T_{i}^{4}\right)+\right. \\
& +\varepsilon_{1}\left(S_{1} V_{i}\right)_{k}\left(a_{k T_{1}} T_{1}^{4}-a_{k T_{i}} T_{i}^{4}\right) \\
& \left.+\varepsilon_{2}\left(S_{2} V_{i}\right)_{k}\left(a_{k T_{2}} T_{2}^{4}-a_{k T_{i}} T_{i}^{4}\right)\right\}
\end{aligned}
$$

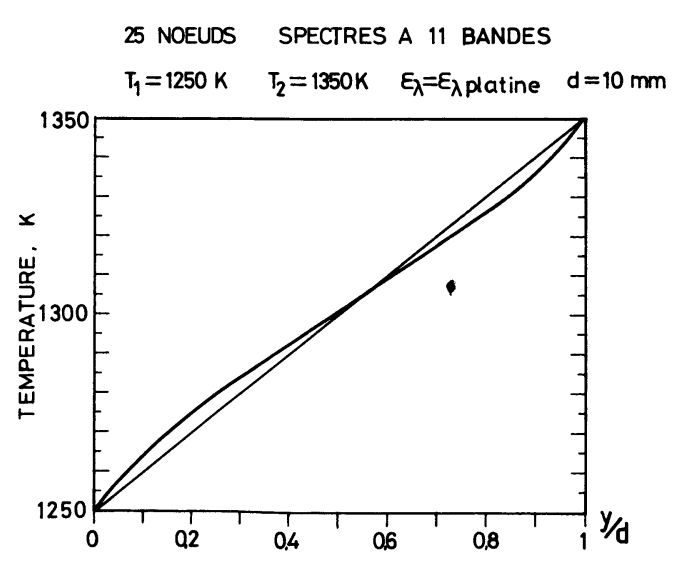

Fig. A.1. - Profil de température établi dans une couche de $10 \mathrm{~mm}$ de $\mathrm{B}_{2} \mathrm{O}_{3}$ avec frontières en platine.

[Temperature profile within a layer of $10 \mathrm{~mm}$ of $\mathrm{B}_{2} \mathrm{O}_{3}$ fitted with platinum boundaries.] 
où les $a_{k T_{i}}$ sont des fractions spectrales sur la bande $k$, et les $\left(S_{i} V_{j}\right)_{k},\left(V_{i} V_{j}\right)_{k}$ sont les aires d'échanges étendus, relatives à la bande spectrale $k$ qui s'expriment au moyen des aires d'échanges directs $\left(s_{i} s_{j}\right)_{k}$ de frontières noires à l'aide des relations de Gebhart [3].

Lorsque (A.3) est introduit dans (A.2), la température du nœud $i$ est donnée par une équation de la forme :

$$
\alpha T_{i}^{4}+\beta T_{i}+\gamma=0
$$

les coefficients $\alpha, \beta, \gamma$ dépendant des différences aires d'échanges étendus ainsi que des températures pour les nœuds $j \neq i$.

Sur la figure A1 le profil de température établi dans une couche de $\mathrm{B}_{2} \mathrm{O}_{3}$ de $10 \mathrm{~mm}$ d'épaisseur, à frontière de platine, a été reporté. On remarque, l'accentuation des gradients thermiques aux bords et la déviation par rapport au profil linéaire du champ de température, ce qui entraîne une répartition non uniforme, séparément, des flux conductif et radiatif comme on a pu le noter sur la figure 8 du texte.

\section{Bibliographie}

[1] PoltZ, H., Die Wärmeleitfahigheit von Flüssigheiten II/Der Strahlungsanteil der effectiven Wärmeleitfahigheit. Int. J. Heat Mass Transfer 8 (1965) 515-527.

[2] ArPaCi, H. C. and Larsen, P. S., A thick gas model near boundaries. AIAA J. 7 (1969) 602-605.

[3] Kunc, T., Lallemand, M. and Saulnier, J. B., Some new development on coupled radiative conductive heat transfer in glasses. Int. J. Heat Mass Transfer 27 (12) 2307-2319.

[4] Kunc, T. et Lallemand, M., Transferts thermiques dans les verres à haute température. Glastech. Berich. 58 (8) (1985) 224-231 ; 58 (9) (1985) 259271.

[5] Asahima, T. et Kosaka, Conductivité thermique des sels fondus. Sels fondus 19 (3) (1976) 279 300 .

[6] Smirnov, M. V. et KhoKhlov, V. A., Structures des solutions ioniques et des électrolytes solides. Institut de Chimie Générale de l'Académie des Sciences de Kiev Nauka Dumka (1977).

[7] SANTiNi, R., TAdrist, L., PANTAloni, J. and CERISIER, P., Measurement of thermal conductivity of Molten Salts in range $100-500{ }^{\circ} \mathrm{C}$. Int. J. Heat Mass Transfer 27 (1984) 623-626.

[8] Tufeu, R., Petitet, J. P., Denielou, L. and Le NEINDRE, B., Experimental Determination of the Thermal Conductivity of Molten Pure Salts and Salt Mixtures, Int. J. Thermophys. 6 (4) (1985) 215-330.

[9] Creffield, G. K. and Wickens, A. J., Thermal conductivity of Anhydrous Borax, Boric Oxide and Sodium Sulfate. J. Chem. Eng. Data 20 (3) (1975) 223-225.

[10] Lezhenin, F. F., Korneitchook, A. A., Berjatit, V. I. and GRITZAENKO, V. P., Thermal conductivity of $\mathrm{B}_{2}^{*} \mathrm{O}_{3}$. Teplofiz. Teplotechn. 25 (1973) 94-96.

[11] Tenney, A. S. and Wong, J., Vibrational Spectra of Vapor-Deposited Binary Borosilicate Glasses. J. Chem. Phys. 56 (11) (1972) 5516-5523.

[12] Greenberg, J. and Hallgren, L. J., Infrared Absorption Spectra of Alkali Metal Nitrates and Nitrites above and below the Melting Point. $J$. Chem. Phys. 33 (3) (1960) 900-902.

[13] Wilmshurlt, J. K., J. Chem. Phys. 35 (1961) 10781084.
[14] BATES, J. B. and BoyD, G. E., Infrared Emission Spectra of Molten Salts. Applied Spectroscopy 27 (3) (1973) 204-208.

[15] KuSABIRAKI, K. and ShIRAISHI, Y., Structural investigation of Molten and Glassy silicate by means of Infrared Emission Spectroscopy. J. Physique, Colloq. 43 (1982) C9-351-354.

[16] Prod'homme, L., Certains aspects de l'évolution thermique et de la stabilisation des propriétés des verres. Rev. Opt. 16 (1957) 309-342, 391-422.

[17] En toute rigueur l'interface air-métal est plus émissive que l'interface liquide-métal, sur la base duquel les calculs de réflectivité $\rho_{\lambda 2}$ ont été effectués à partir des équations de Fresnel. Cependant, compte tenu des incertitudes des données expérimentales sur les constantes optiques $\left(n_{\lambda}^{*}=n_{\lambda}+i k_{\lambda}\right)$ des métaux, notamment du platine, les déterminations du coefficient d'absorption d'après la formule (1) ont été faites à l'aide des valeurs de réflectivité de l'interface vide-métal. Pour l'or, la variation de $\rho_{\lambda 2}$ que l'on peut obtenir est inférieure à l'erreur entre les valeurs calculées et l'expérience et ont été également négligées.

[18] Touloukian, Y. S., Thermal Radiative Properties Metallic Elements. (New York, Plenum Press) 1970.

[19] Broadhead, P. and Newman, J. A., OH Group and hydrogen bonding in some bore-oxygen compounds. Spectrochim. Acta 28A (1972) 19151923.

[20] Gustafsson, S. E. and Karawacki, E., Refractive Index Measurements in Fused $\mathrm{NaNO}_{3}$ and $\mathrm{KNO}_{3}$ by a Modified Thermooptic Technique. Appl. Optics, 14 (5) (1975) 1105-1110.

[21] Hottel, H. C. and SAROFIM, H. C., Radiative Transfer. (New York, Mac Graw Hill) 1967.

[22] Lallemand, M. et Martinet, J., Processus irréversibles dans les transferts radiatifs. Ann. Phys. Fr. 4 (1979) 371-388.

[23] White, L. R. and DAvis, H. T., Thermal conductivity of Molten Alkali Nitrates. J. Chem. Phys. 47 (1967) 5433-5439.

[24] McDonald, J. and Davis, H. T., Thermal conductivity of binary mixtures of alkali nitrates. J. Phys. Chem. 74 (1970) 725-730.

[25] NishimURA, M., Kagaku Kogaku, 36 (6) (1972) 640647. 